\section{AB005. Complications and management following tracheal resection and reconstruction}

\section{Douglas J. Mathisen}

Massachusetts General Hospital, Harvard Medical School, Boston, MA, USA

Correspondence to: Douglas J. Mathisen, MD. Massachusetts General Hospital, Harvard Medical School, Boston, MA 02114, USA. Email: dmathisen@mgh.harvard.edu.

Abstract: Despite improvements on tube design and ICU care, postintubation tracheal stenosis remains a formidable problem. Complications following resection and reconstruction are devastating and oftentimes difficult and complicated to manage. The best way to manage complications in such patients is to avoid the complication in the first place. Each patient must be carefully evaluated. This includes careful radiologic evaluation to try to identify the location, the length, extent and severity of the injury. Some patients may present in extreme respiratory distress while others present with symptoms of shortness of breath and can be managed electively. The successful management of the critical airway is of utmost importance and can avoid complications. Stenoses can be dilated with either rigid scopes or balloons for temporary relief of symptoms. This allows for careful preparation of the patient, evaluation of other conditions, and weaning from steroids. The condition of the airway is vitally important. If there is a great deal of infection, inflammation, secretions or friability, it is imperative to improve the local conditions. Careful attention to detail of the operation is imperative. One must use a familiar reproducible, precise technique, minimize anastomotic tension, preserve blood supply and be familiar with release maneuvers if needed. We prefer an open interrupted anastomotic technique with absorbable sutures, traction sutures to reduce the tension, development of the pre-tracheal plane, flexion of the neck and suprahyoid laryngeal release if needed. Gentle flexion of the neck with the use of a chain stitch to maintain that position is preferred. It is best to determine the status of the airway and the integrity of the anastomosis in the operating room. This can be done upon completion of the anastomosis by deflating the cuff of the endotracheal tube and giving a pressure ventilation to 20 to $30 \mathrm{~cm}$ of water pressure. The presence of a rush of air is reassuring that the structural integrity is good. If that is not achieved bronchoscopy should be done to evaluate for a technical problem or edema. If concern exists, a tracheostomy should be placed. A mini-tracheostomy or standard tracheostomy if concern exists is performed. They should be placed two rings below the anastomosis after having covered the anastomosis with a strap muscle flap. A strap muscle should always be interposed if the innominate artery is in proximity to the tracheostomy tube. Checking for an air leak is done by leaving the cuff down, covering the mouth and nose and insufflating to 20,30 and $40 \mathrm{~cm}$ of water pressure. Any air leak that is present must be repaired before leaving the operating room. If concern exists about the airway at the completion of the operation and no protecting tracheostomy has been placed, one can intubate with a small uncuffed endotracheal tube placed under direct vision over a flexible bronchoscope. Patient should be left intubated for two days, given steroids, diuresis, elevation of the head to allow swelling, fluid overload to resolve. Patient be brought back two days later, extubated under anesthesia and a determination made as to the status of the airway. If there is any question, a formal tracheostomy as previously described should be performed. The most common postoperative complication is anastomotic swelling. This is more common after laryngotracheal resection and high anastomoses. A short course of steroids (24-48 hours), diuresis and elevation of the head of the bed is recommended. Heliox can be given if patients are symptomatic from shortness of breath. If patients present with subcutaneous air, a wound infection, or stridor, one must take this seriously. If in doubt, the patient must be taken to the operating room and the status of the airway determined. If the airway is found to be stable, radiologic evaluation for each of these previously mentioned scenarios would be appropriate. If an air leak has developed, it is best evaluated to determine if there is a leak from the anastomosis or a more serious problem such as dehiscence and impending separation. These are true emergencies. If there is thought to be a small air leak it can be repaired and buttressed with muscle successfully in most cases. If the status of the anastomosis is in question or it has clearly dehisced and separated, one must secure an airway either as a tracheostomy or a T-tube. Stents have a very limited role. In highly selected patients who present with a stable airway and onset of subcutaneous emphysema, superficial wound infection or bronchoscopic evidence of a small 
area of necrosis or limited dehiscence of the anastomosis, hyperbaric oxygenation (HBO) has been used. Our protocol is to treat with saline nebulizers, nebulized tobramycin to reduce local infection and twice daily HBO for 10 days. The patient must have a stable airway and someone in attendance at all times. If that cannot be assured, then one should not take this approach. We have used this in a small select group of patients. Side effects from HBO include hearing loss, perforated eardrums and visual changes. We have used this 25 times with about an $85 \%$ success rate for mostly airway problems. The hyperbaric oxygen stimulates granulation, angiogenesis, early healing and prompt re-epithelialization. It is imperative to be certain the patient has a stable airway prior to HBO. The airway should be reassessed as needed. Delayed stenosis is best managed with serial dilations with either balloon or rigid bronchoscopes. If re-resection is contemplated, it is best to delay for three to four months, allow reaction, inflammation and swelling to subside. Reoperation can be done in highly selected patients. We have recently published our most recent 24-year experience with 392 postintubation tracheal stenosis patients. We compared this to the original report of 502 patients by Grillo. Both series had a very similar incidence of postoperative morbidity of around $32 \%$. Many complications are quite similar. The most common complications were swelling, granulations and a much smaller number of wound infections, subcutaneous emphysema, separation and/or dehiscence. In the most recent series, there were no trachea innominate artery fistulas. This was present in less than five patients in the original series. This problem has been obviated by the recognition that the suture line should not sit on the innominate artery, the innominate artery itself should not be dissected and tissue interposition with strap muscle is needed to cover the anastomosis.

Keywords: Trachea; complications; tracheostomy

doi: 10.21037/shc.2019.AB005

Cite this abstract as: Mathisen DJ. Complications and management following tracheal resection and reconstruction. Shanghai Chest 2019;3:AB005. 